Meta

Journal des traducteurs

Translators' Journal

\title{
Terminologie spécialisée en contexte judiciaire
}

\section{Jean Marc Colletta}

Volume 40, numéro 2, juin 1995

Usages sociaux des termes : théories et terrains

URI : https://id.erudit.org/iderudit/002350ar

DOI : https://doi.org/10.7202/002350ar

Aller au sommaire du numéro

Éditeur(s)

Les Presses de l'Université de Montréal

ISSN

0026-0452 (imprimé)

1492-1421 (numérique)

Découvrir la revue

Citer cet article

Colletta, J. (1995). Terminologie spécialisée en contexte judiciaire. Meta, 40(2), 250-259. https://doi.org/10.7202/002350ar

\section{Résumé de l'article}

La justice pratiquée dans les tribunaux pour enfants est en France largement basée sur des échanges oraux entre les magistrats et les familles. Or l'intervention d'experts auprès de cette juridiction s'accompagne d'une terminologie spécialisée qui, en addition à la terminologie judiciaire, vient obscurcir les propos des professionnels. Outre une présentation des termes spécialisés les plus couramment employés, on s'interrogera ici sur l'importance, les conséquences et les alternatives à ces emplois terminologiques. 


\title{
TERMINOLOGIE SPÉCIALISÉE EN CONTEXTE JUDICIAIRE
}

Jean Marc COLletta

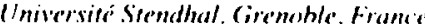

\begin{abstract}
Résumé
La justice pratiquée dans les tribunaux pour enfants est en France largement hasé sur des échanges manx entre les magistrats al les familles. Or l'intervention de cexperts aupres.s

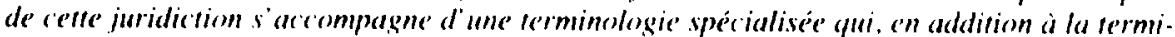

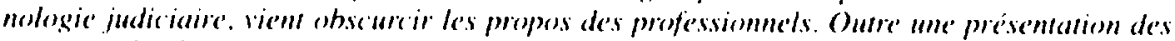

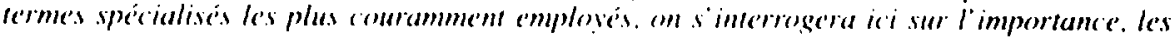
conséquences ef les alternatives à ces emplosis terminologiques.
\end{abstract}

Le terrain judiciaire est, en France, un terrain où les professionnels ont quotidiennement recours à une terminologie spécialisée. On peut ajouter. dans une optique socioterminologique (Gaudin 1993), yue la circulation sociale de centains termes y fait probleme. Quelle est cette terminologie? Comment réagissent les justiciables face à son emploi"? Quels types deffets sont induits par son utilisation? Telles sont les questions auxquelles j'aimerais tenter de répondre, en limitant mon analyse au champ de la juridiction des mincurs.

Less observations qui suivent sont issues d une étude au Tribunal pour Enfants de Grenoble (voir Colletta 1992, chap. 7 à 10), élude qui consistait au départ à décrire et analyser des entretiens entre les juges des enfants et des familles d'origine étrangere afin d'examiner les problèmes d'intercompréhension qui surgissent dans ce type de situations. Les entretiens enregistrés ont pour cadre le cabinet du juge des enfants et consistent en auditions et audiences lors desquelles le magistrat recoit les mineurs et leur famille ainsi que. le cas échéant. les travailleurs sociaux concernés (notamment les éducateurs qui interviennent dans les familles à la demande du juge, ou qui assurent la prise en charge d'un mineur dans une institution de placement).

\section{PRÉCISIONS RELLATIVES A LA JURIDICTION DES MINELIRS}

A la différence des autres juridictions, la juridiction des mineurs présente plusieurs particularités sur lesquelles il convient de s'altarder:

1. Le magistrat de l'enfance est compétent tant en matière pénale (dans le traitement des dossiers de délinquance) qu en matière civile ou dassistance éducative (dans le cadre du dispositif de protection judiciaire de l'enfance en danger). En outre. sa compétence s'étend sur un secteur géographique déterminé. et il intervient à tous les stades de la procédure (saisine, instruction, décision). ce qui permet un suivi des dossiers et des familles par le même juge.

2. Lioralité. le dialogue avec les justiciables, la recherche de solutions négociées constituent des traits caractéristiques de cette juridiction, tant au pénal où le juge des enfants doit s'efforcer d'établir avec le mineur un contrat lengageant dans son avenir. qu'en assistance éducative où, en vertu des lextes, il a pour tâche d'évaluer le degré de danger encouru par le mineur dans son milieu habituel. et doit rechercher l'adhésion de la famille à la mesure envisagée (mesure d'assistance éducative, placement du mineur, ou autre).

3. La pauvreté du droit en matière d'assistance éducative (droit qui ne fournit que des notions vagues: danger. intérêt de l'enfant) rend le magistrat tributaire des experts du champ éducatif qui interviennent aux divers stades de lia procédure et sont en contact 
direct avec les familles et leurs réalités économiques et sociales. Celte caractéristique explique le fait que les juges s'appuient sur les écrits de ces experts (signalements des services sociaux, enquêtes sociales, rapports de comportement, bilans psychologiques...) pour prendre et motiver leurs décisions, et permet d'apprécier l'importance que revêt pour le juge le contact direct avec les justiciables et les experts concernés.

4. La procédure, dans le cabinet des juges des enfants, est moins formelle qu’à l'audience du Tribunal pour Enfants (réservée aux décisions pénales) où tout rappelle les caractéristiques habituelles de la justice. En son cabinet. le magistrat est en lenue de ville, il n'est séparé des justiciables que par son bureau. et le déroulement de l'entretien n'est pas soumis à des règles strictes. (e cadre instaure une plus grande proximite entre magistrats et justiciables et est davantage propice à des échanges de propos spontanés, où la négociation et l"arbitrage ont leur place.

Ces caractéristiques, décrites plus longuement par d'autres auteurs (Amiel et Garapon 1987 ; Lahalle et coll. 1987: Bailleau 1988: Le Roy 1988: Denis 1989), appellent deux commentaires. La justice exercée et rendue au quotidien dans le cabinet des juges des enfants est largement basée sur des échanges oraux entre les protagonistes, et il importe que l'intercompréhension entre les parties soit la plus complète possible. Mais l'intervention d'experts s'accompagne d"une terminologie spécialisée qui. en addition à la terminologie judiciaire, vient obscurcir les propos des professionnels.

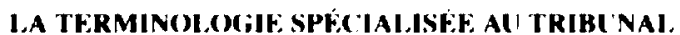

Pour nourrir leur propos, magistrats et travailleurs sociaux puisent dans deux registres

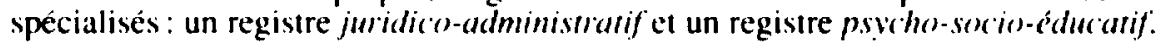

Le registre juridico-administratif est surtout employé par les magistrats. Les termes employés ont pour référent des notions et catégories juridiques, des documents judiciaires et administratifs, des magistrats. des tribunaux. des administrations, des moments de la procédure judiciaire. des mesures civiles et pénales (voir annexe 1, où vont répertoriés les termes les plus fréquemment employés dans notre corpus). (e registre se caractérise par la technicité des termes employés: seuls quelques termes tels "danger". "charges". "instruction», "entendre" sont issus du langage courant et prennent ici un sens juridique.

Le registre psycho-socio-éducatif est au contraire davantage employé par les travailleurs sociaux. If comprend un versant technique, avec des termes dont les référents appartiennent au champ de l'intervention educative: institutions ou organismes sociaux et éducatifs, proféssionnels du champ socio-éducatif. documents rédigés par ces professionnels, modalites d'intervention educative, d'intervention sociale ou d'intervention psychologique (voir annexe 2).

Mais à côté de cette lerminologie technique. on trouve dans le registre psychosocio-éducatif quantité de termes et dexpressions qui font référence soit à des comportements, des attitudes, des états de l'individu ou du groupe familial, soit à des évolutions souhaitables de ces comportements el attitudes, soit encore à des démarches ou des projets de l'équipe éducative auprès des mineurs ou de la famille (voir annexe 3). Les termes employés appartiennent en règle générale au langage courant, mais ils apparaissent dans des expressions où ils perdent leur sens initial. Ainsi le terme "deuil" qui. lorsqu“il est employé dans des expressions telles que "passer au deuil", ne dénote pas l"élat de tristesse causé par le décès d'un proche (sens courant), mais dénote le travail d'élaboration intrapsychique qui succède à la perte de l'objet et permet au sujet de surmonter une phase dépressive (sens psychanalytique. voir Laplanche et Pontalis 1981:5(14).

Il est à ce sujet curieux de constater que ce ne sont pas des psychologues mais des éducateurs qui recourent fréquemment à des termes "psy". Ce recours est peut-être à mettre en rapport avec la construction de l'identité professionnelle de ces derniers. II ne peut en effet s'expliquer totalement ni par la nature des savoirs qu'ils reçoivent pendant leur formation, ni par des habitudes langagières professionnelles, puisque certains éducateurs évitent ce genre d'emprunts au parquet des mineurs. II reste $1^{\circ}$ hypothèse identitaire, que j’ai déjà développée ailleurs (Colletta 1994: 97-98), selon laquelle il y aurait. 
dans ces emprunts au registre psychologique ou psychanalytique, la tentative consciente ou non de la part de l'éducateur de se parer des attributs symboliques des psychologues. soil pour accroître sal légitimité à l'audience. soit pour des raisons identitaires plus profondes.

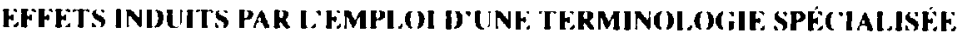

On concevra aisément que le discours spécialisé des juges el des travailleurs sociaux. lorsqu il emprunte à l'un ou à l'autre des deux registres présentés ci-dessus, reste obscur pour le justiciable. Le locuteur qui ne connaît pas les procédures judiciaires. les modalités de l'intervention éducative ou le registre des psychologues ne peut être que désorienté face à des propos aussi lechniques ou hermétiques. Examinons précisément les incidences interactionnelles et discursives de ces emprunts.

Un premier constat : les justiciables réagissent rarement à des propos techniques. Il va de soi que celle absence de réaction n'est en rien l'indice d'une incompréhension de leur part. surtout si les justiciables en question connaissent le parquet des mineurs pour l'avoir déjà fréquenté. Mais il ess troublant de constater que beaucoup d'entre eux ne reprennent jamais la terminologie des professionnels dans leur propre discours, y compris lorsque le juge les incite à donner leur avis (on assiste alors à des réponsés éludées ou à des propos embarrassés). Un exemple illustrera ce type de comportement. Il est extrait d'un dialogue entre une Éducatrice (EI, responsable de Fra. jeune adolescent placé en foyer) el le juge (J):

\section{Extrait $N^{\circ} 1$ *}

El: jecrois que fra it est plein d’bonnes intentions mais c'est vrai qu'c'est difficile de les mettre en

pratique ces bonnes intentions et que: il a besoin qu'on l'aide peut-être à: faire ça - et heu fin nous

c'yu on pensait donc c'élait aussi une mesure d'AEMO mais aussi des: - heu un suivi en CMP - pour

* lui justement

J: $\quad$ mmhm

FI: fin fä c'était sur leconseil de madame XXX donc la psychiatre du foyer - mais jepense qu ce'ese

.. important pour lui

$\mathrm{J}$ : ben écoutes - jovis faire une petite note à: la consultation ps que bon la mesure avait ić déjài

.. ordonné dans l'même temps qu le placement provisoire puisque - éest fá aurait pu permett' de

domer unc premiere indication heu bon - el jvais leur faire une petite note - et puis heu: $\mathrm{j}$ " vont

quand même faire leur travail f̧a permettra d'orienter la famille vers f̧a - hein bon l'AEMOj jense

. $\quad$ qu'elfectivement $\mid /$... soupir ... | elle interviendra: un jour ou l'aut mais c'est pas lat peine

El: $\quad$ |oui c'est vraiment nécessaire

J: d'avoir deux mesures yui s'chevauchent

* Lal transcription est orthographique. L'apostrophe (j'crois) signale une élision : les deux points (que:) un allongement de la syllabe: les crochets I un chevauchement de parole: l'accolade / f un commentaire du transcripteur; le tiret - une pause. qui, si elle est longue, apparaît avec dess séries de deux points entre les tirets $(-;: ;-=3$ secondes $)$.

On remarquera que Fra et ses parents sont doublement exclus de cet échange : ils en sont exclus de par la teneur très technique du discours, et ils en sont exclus énonciativement puisqu'à aucun moment de ce dialogue ils ne sont appelés à prendre la parole. Or 
au cours de cet épisode, $\mathrm{E} l$ «négocie» avec le juge la mise en route d'une mesure d'AEMO dans la famille (c'est-à-dire d'un suivi de la famille par un éducateur) el d'un suivi en Centre Médico-Psychologique pour le mineur, ce qui intéresse au premier chef Fra et ses parents. Pourtant, nulle information relative à cet aspect ne perce jusqu'à la clôture de l'audience, et les parents ne demandent aucune précision, alors même que la terminologie employée interdit de penser, au regard de leurs pratiques langagières (les parents sont Portugais et montrent des difficultés d'él(ccution en français), qu ils aient pu saisir le sens de cette négociation entre le juge et l'éducatrice.

On peut bien sûr s’interroger sur cette réserve des justiciables (le cas présenté ici $n^{\circ}$ en est qu'un parmi bien d'autres). Il me semble qu'elle tient à la nature dissymétrique (doublement dissymétrique pour les familles d'origine étrangère, qui ont de surcroît un handicap linguistique) de leur relation aux professionnels. N'oublions pas que l'audience est avant tout une situation formelle (même si son déroulement ne l'est pas) et que le meneur institué et légitime de l'entretien est le juge. Manifester son incompréhension à ses propos ou aux propos d'un expert n'est pas innocent sur les plans relationnel et identitaire, soit parce que cest implicitement remettre en question la manière dont le professionnel s'exprime, soit à l'inverse pour le justiciable parce que $c$ 'est accentuer sa posilion d'infériorité en exhibant sa propre incompétence linguistique.

Mais les difficultés de compréhension des justiciables apparaissent parfois au grand jour. Lors d'une audience (dont je ne peux reproduire ici les extraits comple tenu de leur longueur. mais qu'on trouvera dans Colletta 1992: 261-266) un père réclame la mainlevée du placement en famille d’accueil de ses deux enfants : de leur côté. les travailleurs sociaux font part de leurs réticences et annoncent leur désir de voir le placement se maintenir. pour diverses raisons. Lun d'entre eux Evoque ensuite la possibilité d'une mainlevée du placement. à condition que le père sengage aussitôt à demander auprès de l'Aide Sociale à l'Enfance une "mesure de recueil lemporaire" de ses enfants. Dans les faits, cette modification ne changerait rien puisque les entants demeureraient une autre année dans leur famille d'accueil. Mais en droit, le père recouvrerait l'autorité parentale et sés enfants ne seraient plus placés par le juge, mais par lui-même, sur sa propre demande. le temps que les conditions d'un retour en famille s'améliorent. Cette proposition Irouve sa logique dans une démarche de responsabilisation du père et de retour progressif à une situation familiale normale (l'issue de la mesure de recueil temporaire étant que les enfants retoument vivre au foyer parental), mais sa subtilité apparaît d'embléc, tant il est vrai qu'elle n'apporte de modifications qu'au seul niveau juridique. Aussi peut-on comprendre qu il ait fallu près de 4.5 min à ce père. né au Maghrèb et peu scolarisé. pour en comprendre la signification exacte.

La présentation qui précède pourrait faire croire que le discours tenu par les professionnels au parquet des mineurs est généralement obscur pour les justiciables. Il convient de modérer celte image. d'une part parce que les professionnels n'empruntent pas en permanence aux registres spécialisés. ensuite parce yue certains justiciables connaisisent approximativement (et dans les meilleurs des cas utilisent) ces registres, enfin parce yue d autres n'hésitent pas, en ce qui concerne surtout les décisions prises par les juges, a faire montre de leur incompréhension. Lorsque c'est le cas, les juges sont donc amenés à reformuler leurs propos.

L'examen de ces reformulations montre cependant qu elles vont de pair avec une présentation simplifiée. elliptique de la mesure envisagée. Prenons un exemple: pour décrire une mesure d'assistance éducative à un père d'origine turque. un juge explique qu il s'agira d'un "soutien du service éducatif... une aide extérieure pour vos problèmes (familiaux) el pour léducation de vos enfants": un peu plus tard et a la demande du père. il précise que cela "consistera à désigner un éducateur qui assisfe. qui soutient, qui aide les parents à vivre cette situation pour éviter des problemes aux enfants". Après une nouvelle demande de précision du père. le juge ajoute qu il s'agira d"une "aide éducative à domicile». On remarquera le manque de précisions concrètes apportées par le juge. qui sen tient à des formulations vagues (un éducateur qui assiste, qui soutient, qui aide). manque de précisions qui est sans doute a lorigine de cette remarque ultérieure du père à 
l'interprète présent lors de l'audience: «Mais qu'est ce qu'ils (le service éducatif) vont venir faire chez moi? ils vont quand même pas venir faire à manger!»

Cette remarque est intéressante à plus d'un titre. D'une part elle montre que les explications des juges et des travailleurs sociaux, lorsqu'elles demeurent vagues et abstraites, ne permettent pas de contourner l'écueil terminologique. $\mathrm{D}$ 'autre part. elle montre combien il est difficile pour les justiciables de se construire des représentations adéquates des mesures prises par les juges, a fortiori s'ils sont d'origine étrangère et viennent de pays culturellement et institutionnellement éloignés du nôtre. Tout se passe comme si, par-delà la barrière terminologique entre professionnels el justiciables, se dressait une barrière culturelle.

Il arrive enfin que le discours spécialisé, lorsqu'il emprunte au registre psychosocio-éducatif, suscite des réactions de la part des justiciables. Ainsi dans l'exemple ci-après:

\section{Extrail No2}

F2 ess l'éducatrice chargée de Am, jeune fille de 14 ans, au foyer où cêlle-ci est placée ; elle Everyuc les intentions du foyer à son égard :

E2: c'est dans c'sens la qu'on attend - de la part d'Am - un: accrochage - à c'qui lui est propose - -:-

qu elle puisse s'accrocher a cette prise en charge qui devient différente - hein bon on ta posé des

" limites on a dit tu vas rentrer a telle heure et cetera - bon maintenant - on a cenvie d"passer à

." aut chose Am - à seize ans bon heu - t'es une jeune tu - tu es capab de nous montrer que tu as des

- et cá alors

compontements - tout à fait - mais vraiment très très chouettes dans l'cad de ton travail

. heu: - impeccables - mais à d'aut'moments - heu tu as du mal à t'maîtriser - et c'est là-dessus yuion

- a a travailler sur cette maitrise de toi - et sur la souffirance que tu as - d'événements el dont tu ne

". peux pas parler -:.:.:- et: bon heu j"pense que: on a élé clairs la "rnière fois qu 'on s'est rencontré - si tu

" ne parles pas d'ces événements qui sont là - heu - on n’ pourra pas passer au deuil tu réagiras

“ toujours de façon impulsive - et en posant des acles - c'est important pour nous que tu arrives à

" passer par - la: la parole

Am : -::- mais: vous vous dites ouajs: l'as - ca t travailles mais en fait fa m travaille pas hein - non: c'est

" vrai: ch'pas vous m dites que - ch'fais des choses mais s' -......-

E2 : que quoi titravailles

Am : sur ćyui s'est passé sur mon frère là - pas longtemps - moi ça m fait rien du tout

Le discours de E2, à l'instar de celui des psychologues, objective le vécu psychologique ou relationnel d'un justiciable el a pour effet d'imposer une vision externe sur des processus internes, de proposer une définition d'autrui sur laquelle celui-ci n'a guère de prise. Si ce type de discours est accepté voire attendu dans la relation thérapeutique, il n'est pas sans violence symbolique dans une telle situation. De là vient sans doute le fait que face à ce type de discours, les justiciables prennent parfois des positions divergentes (dénégation, réfutation, distance dans les enchainements discursifs). comme Am dans le dernier extrait. Les propos de la jeune fille montrent d ailleurs que celle-ci a parfaitement compris le sens de l'intervention de son éducatrice, méme si la signification de certains termes a pu lui échapper. 


\section{POUR CONCLIURE}

Au vu de la présente élude, il serait souhaitable que les professionnels du judiciaire et de l'éducatif essaient de mieux adapter leurs propos au public. soit en restreignant leur usage de termes techniques ou dexpressions spécialisées, soit lorsque ce neest pas possible (à tout le moins le juge est-il contraint de nommer les mesures qu il prend). en reformulant leurs propos atin de les rendre plus explicites. Un tel changement de comportement requiert au préalable une sensibilisation des professionnels, par exemple à travers des démarches de formation continue. comme je lai proposé antérieurement (Colletta 1992. chap. 9). Sur un versant plus directement linguistique se pose la question de la pertinence de la terminologie juridique et administrative actuelle. compte tenu des problèmes d'accessibilité et de lisibilité qu elle induit che le public de la juridiction de lenfance. La direction de la Protection Judiciaire de la Jeunesse est consciente de ces problèmes puisqu une recherche est actuelkement en cours a Grenoble, sous la direction de C. Blattier.

Mais les difficultés de compréhension entre justiciables el professionnels ne tiennent pas qu’à des considérations de nalure linguistique ou sociolinguistique : elles tiennent aussi. on l'a vu. à des considérations de nature culturelle el idéologique. Par ailleurs. comme le montrent les réactions négatives de certains justiciables. il y a un écart entre comprendre les propositions ou les explications d'autrui, même lorsqu elles sont partiellement dissimulées derrière l'écran terminologique, et les admettre. Én conséquence, si les problèmes posés par l'emploi d'une terminologie spécialisée au parquet des mineurs ne peuvent être ignorés, ils doivent être remis à leur juste place car des problèmes plus aigus demeurent. concernant la justification par les institutions judiciaire et éducative des modalités d'intervention éducative et psychologique ou des mesures de placement auprès des familles concernées. Est-ce un hasard si, aux dires des juges pour enfants eux-mémes, les dossiers d'assistance éducative sont plus délicats à traiter que les dossiers de délinquance?

\section{RÉFERENCES}

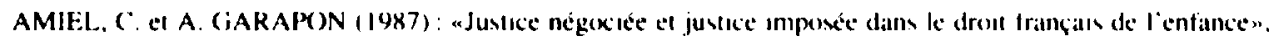
Anmales de Lam resum, n" 2, Vaucresson, pp. 19-42.

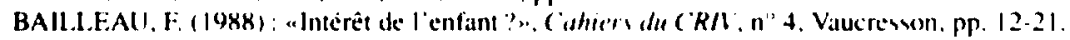

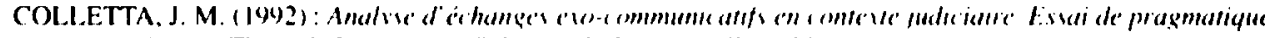
interpreifarive. The ne de Dextorat en Sciences du langage. (irenoble.

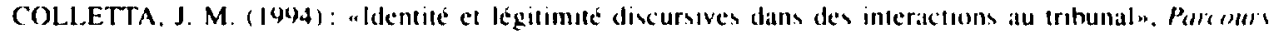

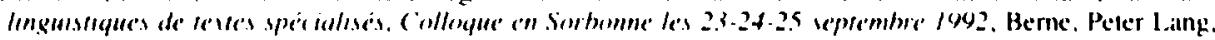
pp. $87-49$.

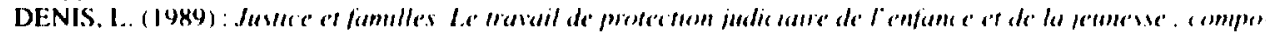

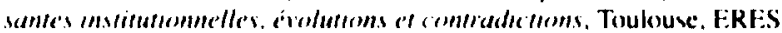

GAUDIN, F. ( 1993$)$ : Peme ane soc meremimolegre. Rouen. Publications de I'Universite de Rouen.

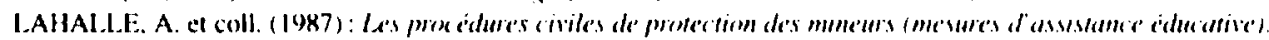

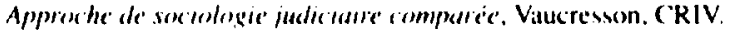

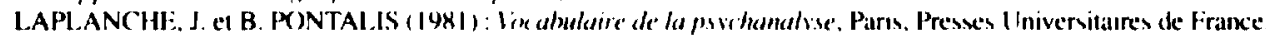

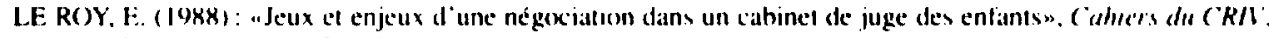
n"4. Vaucresion. pp. 134.146.

\section{ANNEXE: 1}

\section{Répertoire des termes les plus courants du reglstre juridico-administratif}

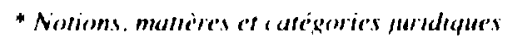

\section{termes}

- assistance éducative

autorité parentale

- conclusions au fond

danger

a demandeur d'asile

\section{contexles d'utilisation}

$\Rightarrow$ en matière $d^{*}$... : decider si une ... est nécessaire

$\Rightarrow$ le titulaure de $l^{\prime} \ldots: l^{\prime} \ldots$ a eté confice ì : promonecr une

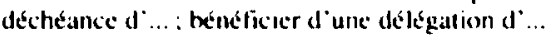

=> dans l'altente des... de Mme X

$\Rightarrow$ en ituation de ... : apprecier le ... ches 
- devorrs. charges

- aroil

- droit de garde. de visite, d'hébergement

- enfant légitime, enfiant naturel

- execution

- garde. garde conjointe

- gardicnnc

- intćrèt de l'entant

- minorite, majorite

Dartices

- prexedure (civile. póniales

- protection judiciaire des jeunes majeurs ou P.J.J.

- régime

- tutelle d lita

- vice de princidure
$=>$. attakhéle) à un droit

$\Rightarrow$... fixe (ou exerce) a l amiable

$=>$ fixer le ... : exercer un ... : le ... es suspendu

$=>$ apprécerer l'... d'un droil $:$ des difficultén d'..

$\Rightarrow$ actucllement. qui excerce la ...?

$\Rightarrow$ Mme X est lat ... de lientiant

$\Rightarrow$ négexier dans l'... intervenur sur le terran de l"...

$=>$ dans le ciadre de sal ...

$\Rightarrow$ athutir a un accord entre les...

$\Rightarrow$ dans le cadre de lat ... d instruction : un incident de ..

$\Rightarrow \quad$ dams le calde de la ..

$\Rightarrow$ lomber sous le meime ... de détention que les majeurs

$=>$ on in demandi pour lui une.

$=>$ il ny a a eu atucun ... dalls ce dowier

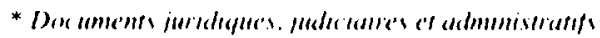

a code civil, pénal, cexde de procédure civile

- comple rendu

- dosvier

- ordonnance, ordonnance de placement.

de renvoi, de non-concilation.

ordonnance de placement provisoire ou ().P.P.

ordomnance juridictomnelle

- proces verbal ou P.V.

$=>$ au regard du ... : f̧a n apparaît pas dans le ...

$=>\ldots$ d audience

$\Rightarrow$ le ... d'asistance Educaltuve: a a vu dev éléments du ..

$=>$ rendre unk ... : I"... du juge $x$
$=>$ Jo vous donne lecture du ... de saisic, d'audition. d'audience

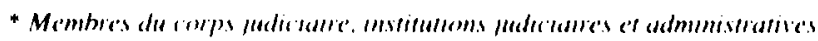

- assessecurs du Tribunal pour Enfants

- avocal

- brigade des mincurs

- chambre de la famille

grefticr

nuinsicer

- juge des enfants, juge des tutelles, juge aux affaires matrimoniales ou J.A.M., juge d'instruction. juge de la mise en état (de la chambre de)

- precureur de la répuhlique, substitut du procureur

- préfecture, OFPRA...

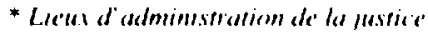

- greffe

- imaison d arrêt

- Tribunal pour Enfants. Tribunal Correctionnel. Tribunal de Grande Instance. Cour d'Appel, ...

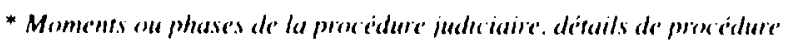

- audition, audience

a decivion

- déxigner, mandater, nommer

- dispositions

centendre

anstruction

Dintervenir

- jugement
$\Rightarrow$ a l'... du : nous nous reverrons a l'...

$=>$ prendre une .... motıver la ... : lia ... est annulee par: aboutir à une ... négeciéce : une ... fixant le droit de visite à $\Rightarrow$ je vais ... un éducateur. ... un service

$\Rightarrow$ reconduire les ... de l'ordonnance du

$\Rightarrow j^{\circ}$ penrrais 1 , an autre jour:

lorsque ge vous ai ... la demière fois

=> nous nous reverron, à $\mathrm{I}^{\circ}$...

$=>$ je dois décider, il est légitime $d^{*}$... dans ce dossier: ... au vu des éléments nouveaux

$=>$ rendre un ... : le ... rendu le 
- mesure, mesure éducative. mesure de garde notification

poursuites

- première comparution ou première compa

- requéte

- saisine. signalement

- se saisir de

statuer

* Decisions el mesures pénales ef civiles

- Action F́ducative en Milieu Ouver ou A.E.M.O. arrêt

- Consultation d'Orientation Educative ou C.O.A.E.

- enquête. enquête sociale

- liberté surveillé, mise en liberté

Observation en Milieu Ouvert ou O.M.O.

- placement, placement provisoire, définitif

retour en famille

- sursis (avec) mise à l'épreuve
$=>$ ordonner une ... : renouveler la ... : reconduire une ...

$\Rightarrow>$ la ... de la decision : la ... de rejet a élé prise par

$\Rightarrow$ engager des ... pénales

$\Rightarrow>$ a quand remonte la ...?

$=>$ introduire une $\ldots$ en divorce :

demander une ... au juge des tutelles

$\Rightarrow$ d'apres le ... des services sociaux

$\Rightarrow$ le juge des tutelles refuse de ... du dossier

$\Rightarrow$ le juge ... au terme de : je dois ... sur la garde de

=> engager une ... : le rapport d'... signale que

$\Rightarrow$ en vertu de l'... rendu par la Cour d'Appel

$\Rightarrow$ ordonner une

$\Rightarrow$ ordonner une ...; reprendre les conclusions de I'...

=> tu as déjà bénéficié d'une ... il me semble

$\Rightarrow$ une ... avait elé ordonnce par

$\Rightarrow$ préparer un ... : ordonner un

le responsable du ... ce ... a une durée legale de

la mainlevée du ... : un ... à la DDASS, à l'Aide Sociale

a l'enfance, en famille d'accueil, en foyer

$=>$ envisager un ... : un ... se prépare

\section{ANNEXE 2}

Repertoire des termes les plus courants du reglstre psycho-socio-educatif (termes techniques)

* Instifutions ef aganismes soc iaus el educatifs

\section{termes}

Acion Sanitaire et Sociale ou DDASS

- Aide Sociale à I'Enfance ou A.S.E.

- Centre Médico-Psychologique ou C.M.P.

- DISS (= Dir. de l'lisère a la Santé el

à la Solidarité)

- E.R.E.A. ( = Etbmt. Régional d'Educ. adaptée)

- foyer, foyer d'accucil. foyer de l'enfance

- services sociaux.

- service de la Sauvegarde. du Codase

\section{contexter d'utilisation}

$\Rightarrow$ il faut faire une demande à l".

$\Rightarrow$ le rapport de l'... signale yue

$\Rightarrow>$ il est actucllement suivi au ... de

$\Rightarrow$ le rapport de la ... signale que

$=>$ il est scolarisé à l"... de

$\Rightarrow$ elle est actuellement placée au ... X: un placement en ..

s impose : l'enciadrement du ... reste le même

$=>$ le signalement des... fait étal de

$\Rightarrow$ le ... mia alerté sur la situation de voure famille

* Professiommels du champ socolo-ćdicuatif

assistante sociale, A.S. de secteur

- educateur(trice)

- inspecteur(trice ) de l'Aide Sociale a l'Enfance

psychologue ou piy

- puericultrice, travalleuse familiale...

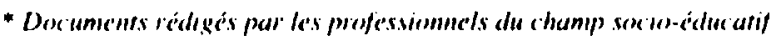

C hilan

- conclusions

- rapport
$\Rightarrow$... de comportement: ... de l'AEMO: vous seres convouués pour un ... à la rentrét

$\Rightarrow>$ les... de l'enquête sociale, de l'entretien psychologique. du bilan AEMO

$\Rightarrow>$... de comportement :... d'enquète siciale 


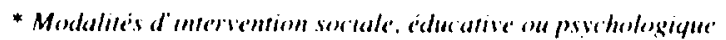

- consultation

- entretiens familiaux, individuels.

$=>\ldots$ ordennéce par

piychothérapeutiques

- examen piychologryue.

$\Rightarrow$ mettre en plase des...

expertise puychologique, médicale

- imervention éducative

- mesure de recueil temperaire ou R.T.

- orrentation

- prise en charge

$\Rightarrow$ fare une demande d'...

$\Rightarrow>$ je dois décider d'une ... dans votre famille

$\Rightarrow$ envisiger une demande de ... a l'A.S.F.

$\Rightarrow>$ nous sommes plutôt pour une ... en foyer

$\Rightarrow$ dim le cadre de la ...: ... éducative. psychologique, linancitere

- suivi

$=>$... du muneur, de lit famille: ... en foyer, en milieu ouverr: faire un ... régulser. à domicile: mettre cen plices un ...

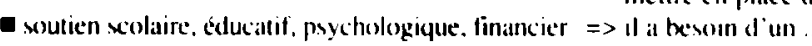

- surveillance médrcale

$\Rightarrow$ il est nécessaire que cet enfant reçoive une ...

eravail

=> latre un ... Élucatif.

therapeutique:

le ... mene par len educateurs (l"iquipe)

\section{ANNEXE: 3}

Répertoire des termes les plus courants du registre psycho-socio-éducatif (lermes non techniques)

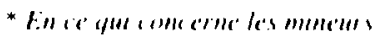

\section{termes}

- hlecage. êrre blexpue

- carences affectives

- comporremente décheco

a conflit. rivallits.

- crive

- difficultes, problemes, troubles

Echec volaure

Denfant symbole.

- enfalll symptòmc

- fugues

- hors réallik

a investur, sinvestur

a mvestussement

anju

- perte affectivic

- pasage à l'acke pamer à l'acke.

amente enacte

a poser des actes

- phétopalhice relard ponderall, de crovisance.

puychomoteur

- relations

- se repirer. x stuer

- sentiments de culpabilite

- souflance. detresse paychologique

- subar cles valconces tratumbalisantes

- Ientialive de sulcide

- Iranseresser la lol

\section{contextes d'utilisation}

$=>$ il est ... dans son ivolution

$=>$ cet enfarm a commu d importintes ...

$\Rightarrow$ on note des... répeites

$=>$ Il est en ... tries gralnde aves son pire

$=>$ etre en ... . entant sujet at des ... d'angoise

$=>$ scolates. de compretement. de persommalite.

prychologicues. relatuomes. de communication. de dialoreute

$\Rightarrow$ elle al cte mise en position d"..

$=>c^{\circ} \times d l^{\circ}$... de la famille

$=>$ il est trop ...

$=>$ clle est tres ... par ses parents depuss son plus jeune age: : Il sest ... dams cette relalion

$=>$ altutude reactionmelle a un ... affectsf trop important

$\Rightarrow$ elle fail lohjet diun ... mportant entre sa mere et a tante

$\Rightarrow>$ lom de la separalion des parents, elle a suhi une importiante...

$\Rightarrow$ Il $y$ a $\mathrm{s}$ (ll ...

$\Rightarrow$ clle a ... les cratinters de sers paremts

$=>$ il al quand meme ... qui mentrent que

$\Rightarrow$ l'enfint presente un important

$=>11$ a des ... perturbees avece les autres membres de sil famille : elle a une ... lusiomelle avec son pere

=> Il a du mal a ... par rapport a lat lou

$=>$ il eprouve dev.... a i l "equard de

$\Rightarrow$ citre dath tll statt de.

$=>$ tres keune. II a .

$\Rightarrow$ au toyer. 11 at ... a plusicurs represes 


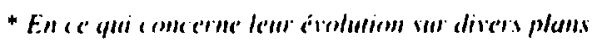

- attitude. componement

- cudre

- identité. ego

- limiles

- passer au deuil

- passer par la parole

a règles, norme

a siluer, se structurer, se repérer

averhaliver ses problèmes
=> il doil changer de ...

=> avoir un ... : se fixer dans un ...

$\Rightarrow$ elle a a construire son .... la reconsiruction de son ...

$\Rightarrow$ avorr des.... il a besoin de ...

$\Rightarrow$ tu ne pourras pas... "l tu ne parles pas de

=> il faul que tu arrives à ...

$\Rightarrow$ il doit respecter les..., rester dans la ...

=> il a besoin de ... : se ... par rapport à la loi

* Ein cre yar comiene les parems. la famille

- alcoolisme

- attitude possessive

- autorité

- hesoin d une tierce personne.

de quelyu un d'extérieur

- contexte familaal

- depression

non-dils

pressions familiales

- prohlemes de couples. de relations

\section{véalité}

- tentative de suicide

- violences, sévices

$\Rightarrow 1^{\prime} \ldots$ de Mme $X$ est ressentie de façon tres negative $\Rightarrow$ le père a un devoir d'... sur ses enfants

=> la famille a ... qui l'aide à surmonter ces difficultés,

$=>$ jaimerals revenır sur le $\ldots$

$\Rightarrow$ des éposodes de ... : la mère est dépressive

$\Rightarrow$ il y a des ... dans la famille

$\Rightarrow>$ les... sont telles que

$\Rightarrow$ les ... sonı bien connus dans cette famille

=> ces éléments de ... : les parents ont été submergés par la ... du comportement de $\mathrm{X}$

* En ce qui conce'ine une evolunion de la famille ond des relations faniliales

- autonomie educative

- cadre familial

exprimer

a liens

réalité

- relations, rapports

- se remeltre en question
$=>$ on doit permettre à la mère de retrouver une ...

$\Rightarrow>$ le ... est à reconsiruire

$\Rightarrow$ pour que vous arrivies à ... ce qu'il en est de voure rapport a

=> il est important de maintenir des ... avec cet enfant

$=>$ il faut élever les enfants dans la ...

$=>$ il faut arriver ì des... normales

=> ils ont du mal à se...

* En ce qui concerne les projets au le vavail emisagé par léquipé edu ative

accompagner

cidre

demande

Démarche

differenciation

Dindividuation

- lieu

metre en place

- poser des limites
$\Rightarrow>$ il faut ... la famille dans ce travail

$\Rightarrow$ de manière a reconstruire un ... seccurisant

$\Rightarrow$ au niveau de la ... : tenir compte de la ... de Mme X

$\Rightarrow$ voir quel est le sens de celle ... dans une ... d'accompagnement

$\Rightarrow$ de manière à favoriser la ... de chacun

$\Rightarrow$ favoriser $\mathrm{I}^{*}$... de $\mathrm{X}$

$\Rightarrow$ des entretiens qui se fasient dans un ... neutre

$=>$ il faul ... un suivi régulier

$\Rightarrow$ nous devons lui... 